\title{
33. The Karyological and the G.banding Analyses of a Polistes Male Wasp, Parapolibia indica Saussure (Vespidae, Hymenoptera)
}

\author{
By Hidehiro HoshiBA \\ Daitobunka University, Daiichi High School, Itabashi, Tokyo 175 \\ (Communicated by Sajiro Makino, M. J. A., March 12, 1985)
}

The cytological studies on the wasps (Polistinae) have been worked out by Machida (1934), Paradi (1947), Hung et al. (1981). Among twelve Polistes species so far studied, the chromosome number varied from six ( $P$. jokohamae, Machida, 1934) to thirty three ( $P$. exclamans, Hung et al., 1981).

Hoshiba and Ono (1985) have been reported thirty chromosomes in the male (haploid) of $P$. snelleni, including the early emerging male, and sixty chromosomes in the female (diploid), whereas Machida (1934) observed thirteen chromosomes in the male of this species from the Yokohama district.

However, no cytological study on the Paraporibia species has been reported. In the present paper, the karyotype and the G-banding analyses of a paper wasp, Parapolibia indica Saussure (Polistinae) are to be reported.

Materials and method. The young larvae of Parapolibia indica were collected at Machida-shi, Tokyo in September, 1984.

They were dissected to obtain the testes when they were in the spermatogonial division, then pretreated by the hypotonic solution $(0.4 \% \mathrm{KCl}, 0.01 \%$ colchicine) for $30 \mathrm{~min}$ and fixed in acetic-methanol $(1: 3)$. They were stored at about $-15{ }^{\circ} \mathrm{C}$. The preparations were made by the conventional air-dried method, and stained with the Giemsa solution. Then, after decolouring by dipping in $70 \%$ acetic methanol, the method described by Seabright (1971) was applied for the G-banding.

The nomenclature of the chromosomes followed the system described by Levan et al. (1964).

Results and discussion. The karyotype and the G-banding patterns were shown in Fig. 1 (a, b), and the schematic G-banding pattern of the male (haploid) $P$. indica was illustrated in Fig. 2. The chromosome number of this species was fourteen and they were divided into three groups, i.e., the group A (nos. 1-3, the large metacentrics), the group B (nos. 4-9, submetacentrics), and the group C (nos. 10-14, metacentrics).

The three chromosomes of the group A looked morphologically similar, but the G-banding analysis defined the difference between nos. 1 or 2 and no. 3 . No. 12 has almost entirely been stained by the G-banding method. The G-bandings of most of the other chromosomes appeared near their centromeres. No special chromcsome as found in the Vespine hornets which had the distinct secondary constriction (Hoshiba and Yamamoto, 1985) was recognized.

The chromosome study on these genera has been considered as a useful taxonomic tool (Hung et al., 1981), and the banding analyses will give more information. The further banding analyses are required from the evolutional and/or cytotaxonomical points of view.

Acknowledgements. The author wishes to express his sincere appreciation 


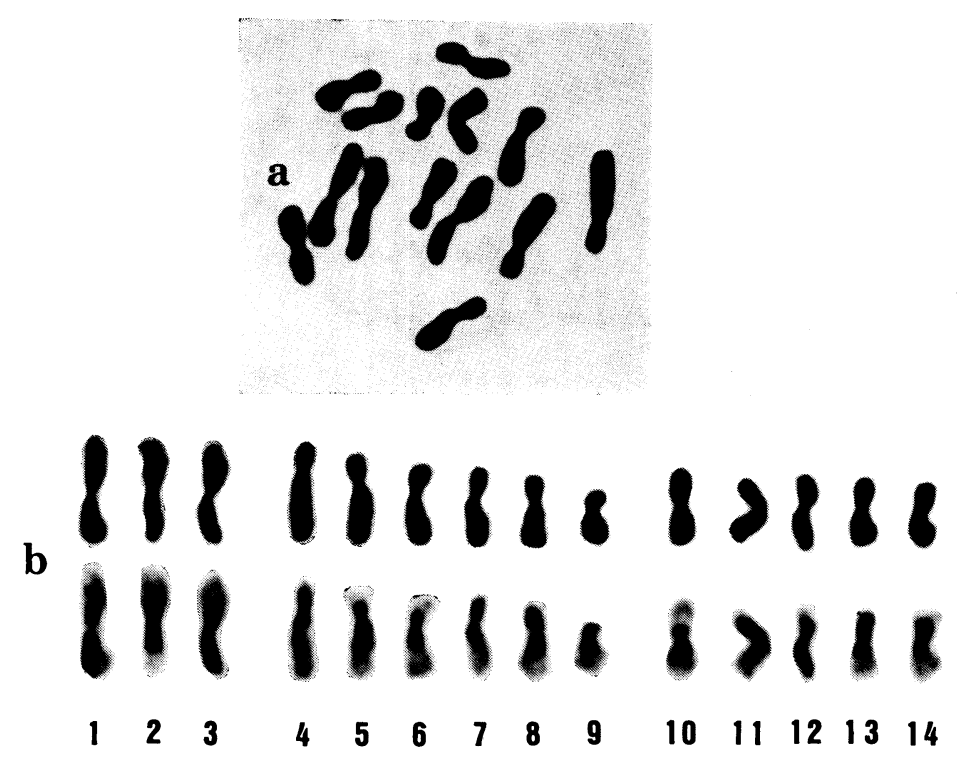

Fig. 1. The meitotic metaphase chromosomes of the male (haploid) Parapolibia indica (a) and its G-banding karyotype pattern (B). Bar indicates $5 \mu \mathrm{m}$.

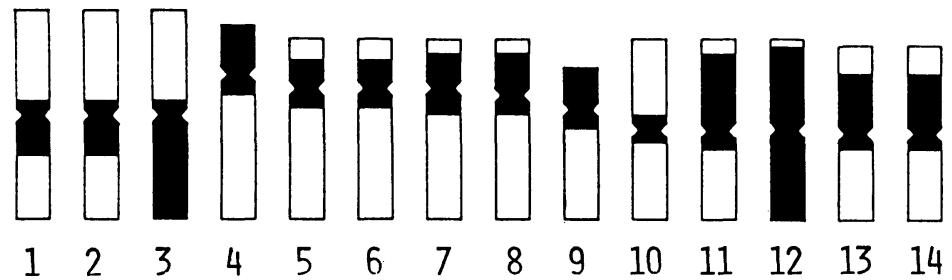

Fig. 2 The schematic G-banding pattern of the male (haploid) Parapolibia indica.

to Professor Emeritus, Dr. S. Makino, M. J. A., of the Hokkaido University for the improvement of this manuscript. He is also greatly indebted to following professors; Dr. I. Okada of the Tamagawa University, Dr. S. Sakai of the Daito Bunka University, and Dr. H. Yamamoto of the Dokkyo University of Medicine.

\section{References}

Hoshiba, H., and M. Ono (1984) : Proc. Japan Acad., 60B, 368-371.

Hoshiba, H., and H. Yamamoto (1985): ibid., 61B, 67-70.

Hung, A. C. F., H. C. Reed, and S. B. Vinson (1981) : Caryologia, 34, 225-230.

Levan, A., K. Fredga, and A. A. Sandberg (1964) : Hereditas, 52, 201-220.

Machida, J. (1934) : Imper. Acad. Japan, 10, 151-158.

Paradi, L. (1947) : Scientia Genetica (Turin), 3, 14-22.

Seabright, M. (1971): Lancet, ii, 971-972. 